Article

\title{
A Distributed Quantum-Behaved Particle Swarm Optimization Using Opposition-Based Learning on Spark for Large-Scale Optimization Problem
}

\author{
Zhaojuan Zhang ${ }^{1}\left(\mathbb{D}\right.$, Wanliang Wang ${ }^{1, * \mathbb{C}}$ and Gaofeng Pan ${ }^{2} \mathbb{D}$ \\ 1 College of Computer Science and Technology, Zhejiang University of Technology, Hangzhou 310023, China; \\ zjzhang@zjut.edu.cn \\ 2 Department of Computer Science and Engineering, University of South Carolina, Columbia, SC 29208, USA; \\ gpan@email.sc.edu \\ * Correspondence: wwl@zjut.edu.cn
}

Received: 30 September 2020; Accepted: 17 October 2020; Published: 23 October 2020

\begin{abstract}
In the era of big data, the size and complexity of the data are increasing especially for those stored in remote locations, and whose difficulty is further increased by the ongoing rapid accumulation of data scale. Real-world optimization problems present new challenges to traditional intelligent optimization algorithms since the traditional serial optimization algorithm has a high computational cost or even cannot deal with it when faced with large-scale distributed data. Responding to these challenges, a distributed cooperative evolutionary algorithm framework using Spark (SDCEA) is first proposed. The SDCEA can be applied to address the challenge due to insufficient computing resources. Second, a distributed quantum-behaved particle swarm optimization algorithm (SDQPSO) based on the SDCEA is proposed, where the opposition-based learning scheme is incorporated to initialize the population, and a parallel search is conducted on distributed spaces. Finally, the performance of the proposed SDQPSO is tested. In comparison with SPSO, SCLPSO, and SALCPSO, SDQPSO can not only improve the search efficiency but also search for a better optimum with almost the same computational cost for the large-scale distributed optimization problem. In conclusion, the proposed SDQPSO based on the SDCEA framework has high scalability, which can be applied to solve the large-scale optimization problem.
\end{abstract}

Keywords: large-scale optimization; spark; qpso; distributed computing; cooperative evolution; opposition-based learning

\section{Introduction}

Nowadays, the development of the Internet of Things has promoted the accumulation of big data. With the increasing complexity of real-world optimization problems, the traditional sequential optimization algorithm may take a long time to complete a task and even cannot deal with it when the data are stored in different places. For the large-scale optimization problem, the accumulation of big data is overwhelming our capacity to analyze through classical techniques, and whose difficulty is further increased by the ongoing rapid accumulation of data scale. This challenge is even greater, especially for the large-scale optimization problem since many local best optimums make the algorithm easily fall into a local optimum, or the computational cost becomes extremely high. However, the traditional optimization algorithm even cannot deal with it when faced with large-scale distributed data. 
Responding to the challenge, distributed optimization is presented as an efficient strategy to improve search efficiency and to reduce this complexity, which can overcome the deficiencies of large-scale, high-dimensional, and complex features in real-world optimization problems. Meanwhile, how to design an efficient distributed optimization algorithm to address such a challenge has received considerable attention over the past decade. Consequently, a distributed framework has become an effective strategy for the big data-driven optimization problem, since distributed computing can improve the search efficiency of the evolutionary algorithm [1,2].

Studies on distributed evolutionary algorithms based on the different distributed frameworks have attracted considerable attention over recent years [3]. In 2004, Google [4] proposed a framework, called MapReduce. As one of the most successful frameworks for distributed computing, it has been widely adopted for analyzing massive, high-dimensional, and dynamic data. Up to date, particle swarm optimization (PSO) plays an essential role in improving the quality of large-scale data, and how to achieve it by distributing is a hot topic. McNabb et al. [5] first proposed a distributed PSO based on MapReduce (MRPSO), which effectively decreases the computational cost. However, MRPSO is not suitable for objectives that are easier to evaluate and is more significant for applications where the computational cost is too expensive.

The distributed optimization algorithm based on MapReduce can address the challenge for the big data-driven optimization problem since the distributed algorithm is better than the non-distributed algorithm concerning convergence speed. However, it takes a long time for MapReduce to deal with computationally intensive operations such as iterative operations, which are especially noticeable if the search space involves a large number of local best positions, or if the computational cost of evaluation becomes very high. Therefore, MapReduce is not a good framework for tasks such as evolutionary algorithms that involve more iterative operations. Conversely, Spark, a distributed computing platform based on Memory, is more capable of iterative computing than MapReduce [6]. As a result, the distributed optimization algorithm based on Spark has attracted considerable attention over the past few years.

Although some studies have been achieved, the distributed optimization by using a distributed framework still requires further research. Therefore, this paper proposes a distributed cooperative evolutionary algorithm framework using Spark (SDCEA). The remainder of this paper is as follows. Section 2 mainly presents the literature review, including real-world optimization applications, large-scale optimization, distributed optimization using MapReduce, and distributed optimization using Spark. Section 3 presents the main steps of the proposed SDCEA, the strategies of the distributed and cooperative co-evolution based on SDCEA. Section 4 presents the methodology, including the initialization and evolution process of the proposed SDQPSO. Section 5 presents the experiment on five typical large-scale optimization test functions and has a discussion on the proposed SDCEA and SDQPSO methodology for further research work. Finally, Section 6 presents the conclusions.

\section{Literature Review}

\subsection{Real-World Optimization Applications}

To our knowledge, recent studies regarding real-world optimization problems remain a challenge faced by current intelligent optimization algorithms.

Ghasemi et al. [7] proposed an uncertain multi-objective model for earthquake evacuation planning. In the proposed model, the total cost of the location-allocation and the amount of the shortage of relief supplies are considered. Furthermore, the proposed model can be applied in a real case study in Tehran in comparison with Non-Dominated Sorting Genetic Algorithm-II (NSGA-II) and epsilon constraint method. The results indicate the superiority of the modified multiple-objective particle swarm optimization (MMOPSO) over the other approaches. 
Later, Ghasemi et al. [8] discussed planning for pre-disaster multi-period location-allocation inventory decisions. A robust simulation-optimization approach is presented to deal with the uncertainty of demand for relief commodities first. Then, a customized genetic algorithm is proposed to solve the resultant deterministic mathematical model. The results indicated that pre-disaster planning can minimize time costs and ultimately reduce the relief time.

For the green closed-loop supply chain under uncertainty problems, modifications of the imperialist competitive algorithm (MICA) can obtain better solutions, which verified the efficiency and performance of the proposed algorithm [9]. Goodarzian et al. [10] discussed how to obtain an optimal solution for multi-objective pharmaceutical supply chain network (PSCN) problems. First, the PSCN problem was formulated as a Mixed-Integer Non-Linear Programming model, and meta-heuristics were selected to deal with uncertainty parameters. After a comparison of meta-heuristics for large-size problems, the results indicated that multi-objective firefly algorithm (MOFFA) can find an acceptable solution efficiently with a relatively low computational cost.

Verdejo et al. [11] proposed a synchronization methodology based particle swarm optimization to tune parameters of power system stabilizers (PSSs) in electric power systems (EPSs). The results on different test systems indicate that it can improve the stability of the large-scale multi-machine electric system.

\subsection{Large-Scale Optimization}

To overcome the several shortcomings, e.g., premature convergence, low accuracy, and poor global searching ability, Zhang et al. [12] proposed a novel simple particle swarm optimization based on random weight and confidence term (SPSORC) for global optimization problems. Later, Yildizdan et al. [13] proposed a hybrid bat algorithm and an artificial bee colony algorithm, and inertia weight was incorporated into the bat algorithm. The result indicates that the hybrid algorithm can produce successful and acceptable results. Furthermore, a new hybrid algorithm, the annealing krill quantum particle swarm optimization (AKQPSO) algorithm, is proposed [14]. The optimized PSO by quantum behavior and optimized KH by simulated annealing show better performance in both exploitation and exploration.

\subsection{Distributed Optimization Using MapReduce}

Traditional optimization algorithms need to be modified to deal with increasing data sizes. Jin (2008) et al. [15] first proposed an improved MapReduce by adding a hierarchical reduction stage to MapReduce. This model is called MRPGA (MapReduce for parallel GAs), which can be adopted to achieve a distributed genetic algorithm. Later, genetic algorithms and distributed algorithms based on the MapReduce framework have been proposed one after another. Wu et al. [16] introduced the divide and conquer strategy and simulated annealing algorithm into the ant colony algorithm (ACO). MapReduce-based ACO was proposed by using a distributed environment of cloud computing, which can improve the efficiency of the solution. Cheng et al. [17] parallelized the ant colony optimization algorithm with dynamic positive and negative feedback based on the Haloop framework built by the MapReduce.

$\mathrm{Xu}$ et al. [18] proposed an improved cuckoo algorithm for the analysis of big data based on MapReduce, which can search for a better optimum at a faster speed. Al-Madi et al. [19] proposed a scalable design and implementation of a glowworm swarm optimization algorithm based on MapReduce, which has a good clustering performance and achieves a very close to linear speedup. Ding et al. [20] divided the whole population into N sub-populations and combined co-evolution and MapReduce to share the results of all independent subsets based on cloud computing, which could obtain a better adaptive balance between search and exploration of solutions.

For the quantum optimization algorithm, Hossain et al. [21] proposed a parallel cluster particle swarm optimization algorithm based on MapReduce by combining PSO and k-means algorithm, which can deal with the problem for service decomposition in the mobile environment by using MapReduce. 
Wang et al. [22] proposed a distributed co-evolutionary particle swarm optimization based on MapReduce, which can shorten the search process of collaborative operation. Further, based on MapReduce, Li et al. [23] and Ding et al. [24] studied the distributed quantum-behaved particle swarm optimization by incorporating the local attractors and quantum rotating gates, and two different encoding and decoding methods. Khalil et al. [25] proposed a MapReduce-based distributed whale algorithm (MR-WOA) to improve the search efficiency for composition space. Furthermore, the speedup scales linearly with increasing the number of computational nodes, which improves the scalability of WOA for solving large-scale problems.

\subsection{Distributed Optimization Using Spark}

For the large-scale global optimization problem, Cao et al. [26] proposed a parallel cooperative particle swarm evolution algorithm combined with GPSO and LPSO by using Spark. Dynamic grouping and multiple calculations are adopted to increase the degree of parallelism, which makes sufficient exploration of the search space and speed up the convergence. Qi et al. [27] proposed a two-stage parallel algorithm, including parallelization of fitness evaluation and parallelization of genetic operations, the results show that the proposed algorithm outperforms the sequential genetic algorithm in both test suite size and computational performance. Liu et al. [28] proposed a parallel GA algorithm based on Spark, which is applied to design optimal deployment schemes for sensor networks. Yuan et al. [29] proposed a Spark-based particle swarm optimization algorithm with dynamic adaptive weight, which is adopted to optimize the input of the K-means algorithm. The results show that the time spent in the execution process can be effectively reduced.

Peng et al. [30] proposed a SparkCUDE algorithm where the Spark with a ring topology by adaptively selecting crossover strategies and the CUDE algorithm is employed as the internal optimizer. The results demonstrate the effectiveness and efficiency of the proposed approach for the large-scale global optimization problem. Later, Teijeiro et al. [31] explored the differential evolution algorithm under different population models, including the master-slave and the island-based DE schemes by using Spark, which shows that the island model has the best performance and shows a decent scalability. Sun et al. [32] proposed a distributed co-evolutionary algorithm for large scale flexible scheduling problems, which divides the data based on sub-problems, and the results show that it has better optimum and lower computational complexity.

\section{A Distributed Cooperative Evolutionary Algorithm Framework Using Spark (SDCEA)}

\subsection{Background of the Distributed Framework}

In recent years, distributed frameworks have been widely used for machine learning, data mining, image processing, and so on. Although the MapReduce is a simple batch computing model, which lacks an effective mechanism for data sharing in various stages of parallel computing, it has been applied for big data-driven optimization. For computationally intensive tasks, e.g., excessive iterative operations, lower computation efficiency can be caused because of the frequently starting map and reduce tasks. To overcome the shortcoming, Spark, a distributed computing framework based on Memory, is proposed by the University of Berkeley. Indeed, Spark is an efficient framework to address the challenge, whose difficulty lies in time consumption for iterative operations.

When faced with big data-driven optimization problems, distributed computing frameworks based on Spark have been proposed since Spark is efficient for evolutionary algorithms evolved through iterative operations. Resilient distributed dataset (RDD) is adopted in Spark, and different operations based on RDD are provided so that distributed data can be processed easily [33]. For dynamic multi-objective optimization problems, Cordero et al. [34] proposed a software package using Spark, called JMetal. Further, Duan et al. [35] proposed a Spark-based software framework for parallel evolutionary algorithms (Spark-based PEAs), and three different particle swarm optimization algorithms are implemented in parallel, which shows that the framework has a high search efficiency 
for computationally expensive optimization problems. Furthermore, the above-mentioned Spark-based software framework can make the algorithm in parallel easier, where JMetal is only proposed for dynamic multi-objective optimization problems, and the Spark-based PEAs are mainly designed concerning software Implementation. Generally speaking, distributed evolution is an effective way for big data-driven optimization problems.

For the large-scale optimization problem, the algorithm may fall into a local optimum easily since there are many local best optimums. Furthermore, the performance of the algorithm is determined by different population distribution and data distribution strategies. Therefore, how to adopt the distributed and parallel framework is a challenge faced by distributed optimization algorithms. In this paper, a distributed cooperative evolutionary algorithm framework using Spark (SDCEA) is proposed, which comprehensively considers computing distribution, population distribution, and data distribution. The SDCEA can greatly improve the performance of computing efficiency while keeping high fault tolerance and high scalability.

\subsection{The Main Steps of the Proposed SDCEA Framework}

The SDCEA framework, as shown in Figure 1, mainly consists of three parts: distributed computation, distributed population, and distributed data. The Spark distributed cluster environment consists of a master node (Master) and multiple slave nodes (Workers), and the computing resource corresponding to the whole workers is a resource pool. More importantly, the distributed computing of the search is achieved by many distributed workers; thus, the computing resource pool is crucial to the performance of algorithms.

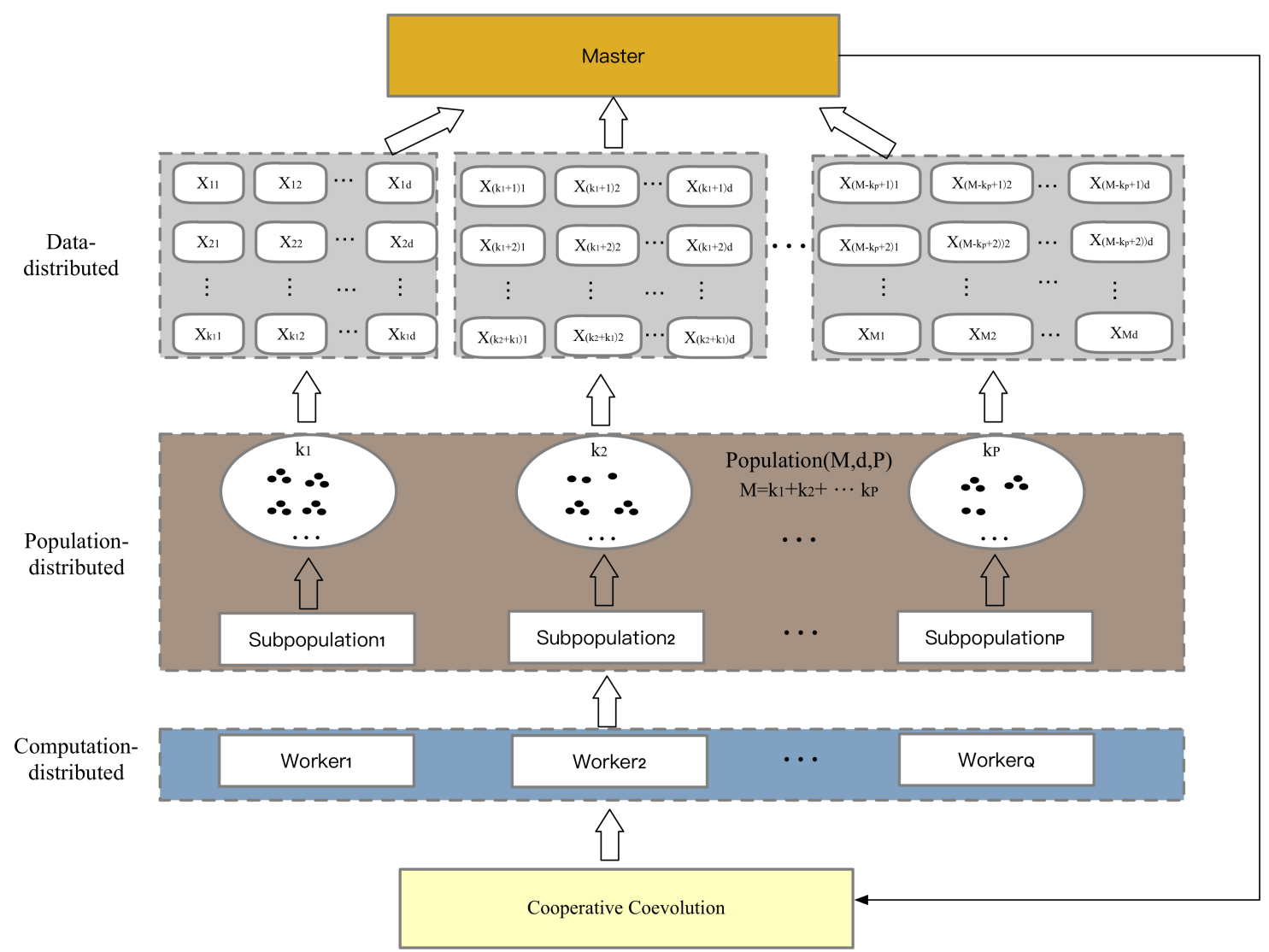

Figure 1. The distributed cooperative evolutionary algorithm framework using Spark (SDCEA) framework.

The SDCEA framework is mainly designed for the evolutionary algorithm that adopts Spark as a distributed computing platform. Furthermore, three distributed strategies are incorporated into the SDCEA framework to address the challenge faced by big data-driven optimization problems. Based on 
this framework, it can be found that the efficiency of distributed computing improved dynamically with the increasing nodes of spark clusters. Therefore, it can be concluded that the SDCEA framework is significant for large-scale optimization problems.

The main steps of the SDCEA framework are given as follows:

1. Configure a distributed computing cluster using Spark. Given $Q$ workers and one master, where $Q$ workers are independent of each other. Based on the task mechanism, the distributed evolution is divided into population distribution and data distribution. Furthermore, the master-slave model is adopted for the proposed SDCEA framework.

2. Group the whole population into many sub-populations based on the parallelization, where each sub-population consists of different individuals, and then each sub-population evolves independently in parallel. Specifically, divide the whole population into $P$ sub-populations denoted by sub-population 1 , sub-population $2, \ldots$, and sub-population $P$, respectively.

3. The search space is partitioned into multiple subspaces for the whole population, and the individual of each sub-population searches on the sub-spaces in parallel.

4. After each sub-population evolves separately for one iteration, the result is further collected through the master. Specifically, the optimum on each search space found by the distributed cooperation is collected by the master. Therefore, the global best optimum of the whole population is achieved, and then the global best optimum is distributed to each worker. Finally, when each worker receives it, the particle position and fitness evaluation of the population are updated.

The above steps 1-4 list a detailed procedure of the SDCEA framework, where each worker searches in parallel first and then cooperate with the master. The master can collect the result of all workers and further compute the global best position of the population at the current iteration. The process is repeated until the search condition is reached, then the whole search process is finished.

\subsection{Distributed and Cooperative Co-evolution Based on SDCEA}

\subsubsection{Population Distribution}

Since distributed evolutionary strategies consist of two main types: population distribution, where individuals of a sub-population are distributed to a different worker, and the same parameter distribution of all sub-populations is adopted; data distribution, where the search space is partitioned into blocks and then is computed in parallel. A distributed evolution model of master-slave cooperative cooperation is proposed for the SDCEA framework, as shown in Figure 2. Furthermore, the population is divided into several sub-populations, and then the distributed evolution of all sub-populations is finished by several workers. Finally, search results are collected by the master among all sub-populations. Consequently, the master obtains the best individual from the workers, and the global best position is sent to all workers.

When populations are divided into several sub-populations, the population is partitioned in parallel based on the degree of parallelism, and each sub-population is independent of each other. Moreover, each subpopulation is evolved according to the update process of the evolutionary algorithm. After one generation, the fitness is recomputed and the global best position of each subpopulation is updated. From Figure 2, where Gbest $t_{P}$ represents the global best position corresponding to the $P$-th subpopulation and Gbest tg $_{g}$ represents the global best position of the whole population, respectively. If the updated fitness of an individual is less than the previous fitness value of the subpopulation, then the fitness value is updated as the global best position of the subpopulation. Furthermore, the individual is selected as the current global best position of the subpopulation for the current generation. 


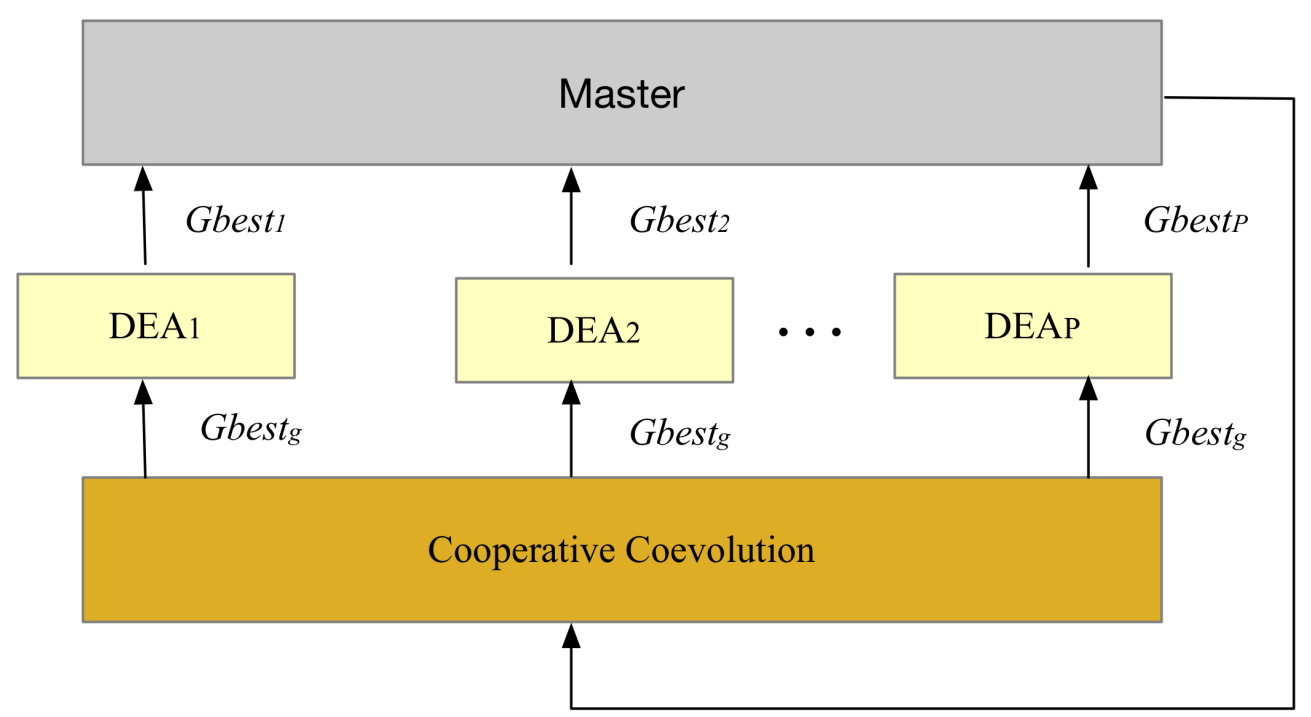

Figure 2. The evolution model of master-slave cooperative cooperation.

\subsubsection{Data Distribution}

For the SDCEA framework, the whole search space is partitioned into several subspaces that evolve independently towards the global best position. The data distribution is illustrated in Figure 3, where the position of particles is parallelized as several subspaces. Furthermore, the performance of the algorithm is much affected by the parallelism of partition. Assuming that the data are parallelized into $P$ groups, namely that the search space can be partitioned into $P$ subspace, and thus the search space is saved as $P$ partitions. The search on each partition is performed serially but in parallel between different partitions. Consequently, data have been parallelized based on the distributed computing framework.

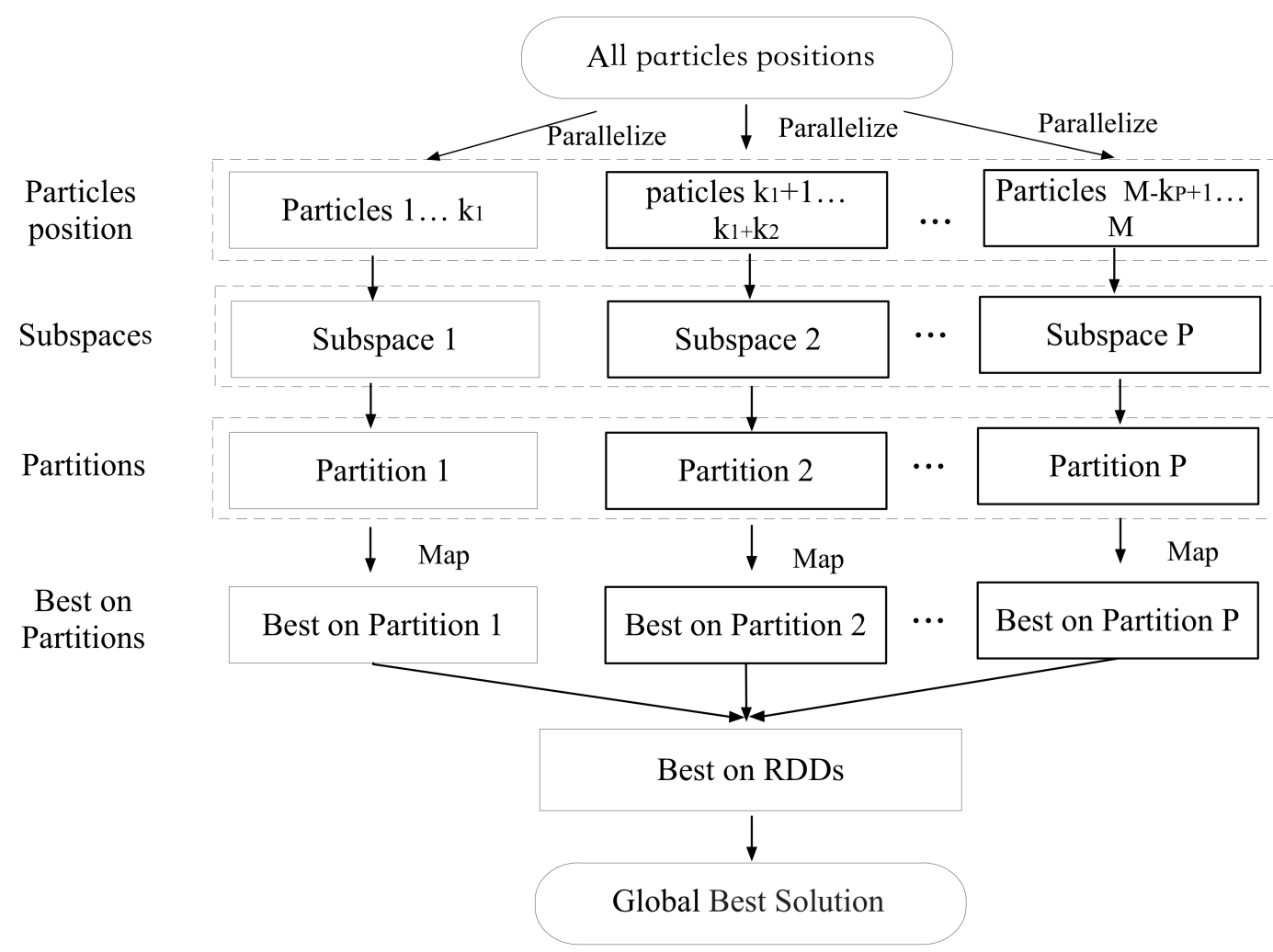

Figure 3. Data distribution of the proposed SDCEA framework. 


\subsubsection{Distributed Cooperative Co-evolution}

For the SDCEA framework, distributed search and cooperative cooperation of the population is achieved based on the Spark. The cooperative co-evolution mainly includes two stages. First, distributed co-evolution is performed independently by all individuals of each sub-population, and each individual concerning all dimensions is updated. Second, further cooperation on the candidate of the global best position is achieved between each worker and the master. Thus, the search of the algorithm is finished through the distributed cooperation of the Spark cluster.

\section{Methodology}

\subsection{SDQPSO Using Opposition-Based Learning}

The QPSO is proposed by introducing quantum physics theory into the particle swarm optimization algorithm, which significantly improves the global search ability of the particle. However, there are still many shortcomings, such as premature phenomena and the diversity of the population decreases through evolving. It is easy to fall into the local optimum if the search space is composed of many local optimums, especially when the data are distributed or stored in different places. To overcome the shortcomings of the QPSO algorithm, a distributed quantum-behaved particle swarm optimization algorithm using spark based on the SDCEA framework is proposed (SDQPSO). The initialization and update process of the SDQPSO algorithm is described below.

\subsubsection{Population Initialization Using Opposition-Based Learning}

The opposition-based learning (OBL) scheme has attracted considerable attention since it was proposed in 2005 [36]. The main idea is to search in the opposite direction of a feasible solution, and then to evaluate the original solution and opposite solution. Finally, find a better solution as the individual for the next generation. The opposite point and opposite solution are defined below.

Definition 1. Opposite point. Let $X=\left(X_{1}, X_{2}, \cdots, X_{d}\right)$ be a point in a d-dimensional space, $X_{i} \in\left[a_{i}, b_{i}\right]$, where $X_{1}, X_{2}, \cdots, X_{d}$ are real numbers, and $i=1,2, \cdots, d$, respectively. Then the opposite point corresponding to $X$ is denoted by $\widetilde{X}=\left(\widetilde{X}_{1}, \widetilde{X}_{2}, \ldots, \widetilde{X}_{d}\right)$, and is given by

$$
\widetilde{X}_{i}=a_{i}+b_{i}-X_{i}
$$

Definition 2. Opposite learning. Given that $X=\left(X_{1}, X_{2}, \cdots, X_{d}\right)$ is a feasible solution of the algorithm and $\widetilde{X}$ is its opposite solution. Let $f(x)$ be the fitness evaluation function, and $f(\widetilde{X})$ is its opposite fitness evaluation function. Then the fitness value is evaluated at the initialization of the population. The learning continues with $\widetilde{X}$ if the fitness value of $f(x)$ is better than the fitness value of $f(\widetilde{X})$, otherwise with $X$.

For the SDQPSO algorithm, the initialization of the population is achieved by adopting the opposition-based learning scheme, which can improve the probability of the population to obtain a better search space and further improve the search efficiency without prior knowledge.

\subsubsection{Evolving through Generations}

The evolution process of the SDQPSO is as follows: first, the master distributes the sub-populations to different workers through the Spark cluster, or the data space has been distributed to each worker; then the update process is performed independently and in parallel on workers. The fitness of each particle is computed and then compared with the global best position on each worker. Then, the personal best position denoted by Pbest is corresponds to each subpopulation, where the personal best position of a particle is the best previous best position (i.e., the position with the best fitness value). Furthermore, the global best position is defined as the best position among 
all the particles, which is denoted by Gbestg. The value of Gbest $t_{g}$ is given by comparing through each sub-population.

The mean best position (mbest) is defined as the mean position of the personal best positions in the population. It is got by the master and sent to each worker, and it is given by

$$
\text { mbest }=\sum_{i=1}^{M} \frac{\text { Pbest }_{i}}{M}=\sum_{i=1}^{M} \frac{\text { Pbest }_{1}}{M}, \sum_{i=1}^{M} \frac{\text { Pbest }_{2}}{M}, \ldots, \sum_{i=1}^{M} \frac{\text { Pbest }_{M}}{M}
$$

where $M$ is the population size, Pbest $_{i}$ represents the personal best position of the $i$-th particle, respectively.

Since the evolution process only considers individual best position and global cooperation, the global best position of each sub-population is considered during the improved evolution process. This can fully reflect the first-stage cooperation among individuals in each sub-population. For each worker, the local attractor is given by

$$
P_{i d}=\mu \text { Pbest }+\frac{(1-\mu)}{2} \text { Gbest }_{j}+\frac{(1-\mu)}{2} \text { Gbest }_{g}
$$

where $P_{i d}$ represents the local attractor of the $i$-th individual, $\mu$ is a random number uniformly distributed on $(0,1)$, and $\mathrm{d}$ is the particle dimension, respectively. Moreover, Gbest ${ }_{j}$ represents the global best position of the $j$-th subpopulation, and Gbest $t_{g}$ represents the global best position of the whole population.

Further, on each worker, the position of each particle in the sub-population is updated generation by generation during the evolution process, and it is given by

$$
X_{i d}(t+1)=\left\{\begin{array}{l}
P_{i d}(t)+\beta \mid \text { mbest }(t)-X_{i d}(t) \mid \times \ln \left(\frac{1}{u}\right), \text { if } u<0.5 \\
P_{i d}(t)-\beta \mid \text { mbest }(t)-X_{i d}(t) \mid \times \ln \left(\frac{1}{u}\right), \text { if } u \geq 0.5
\end{array}\right.
$$

where $X_{i d}(t+1)$ is the current position of the particle in the $t+1$ generation, and $u$ is a random number uniformly distributed on $(0,1)$, respectively. Furthermore, $\beta$ is a contraction-expansion coefficient from 0.5 to 1.0, which is used to control the convergence speed of the particles. Different $\beta$ can affect the convergence speed of the algorithm. Generally, the value of $\beta$ is taken as

$$
\beta=\frac{(1.0-0.5) \times(\text { MAXITER }-t)}{M A X I T E R}+0.5
$$

where MAXITER is the maximum iteration and $t$ represents the current iteration.

Finally, the global best position of each sub-population searched in parallel is collected by the master and the master analysis whether the termination condition is reached. If the termination condition is reached, then the global best position of the whole population is got. Otherwise, the updated parameters, Gbest $g_{g}$ and mbest are distributed to each worker. The whole evolution is repeated until the termination condition is reached, and then the distributed search of the search space based on the SDCEA framework is completed.

\subsection{Evolution Process of the Proposed SDQPSO}

The evolution process of the whole SDQPSO algorithm is shown in Figure 4, where Fes represents the function evaluations, and Max $x_{\text {evals }}$ represents the maximum function evaluations, respectively. The search space saved as RDDs is partitioned into several subspaces, and each sub-population searches in parallel on each worker. The master collects the results of all workers first and then distributes them to the worker. Through the cooperation of the master and each worker, the whole distributed search process is finished when the termination condition is reached. 


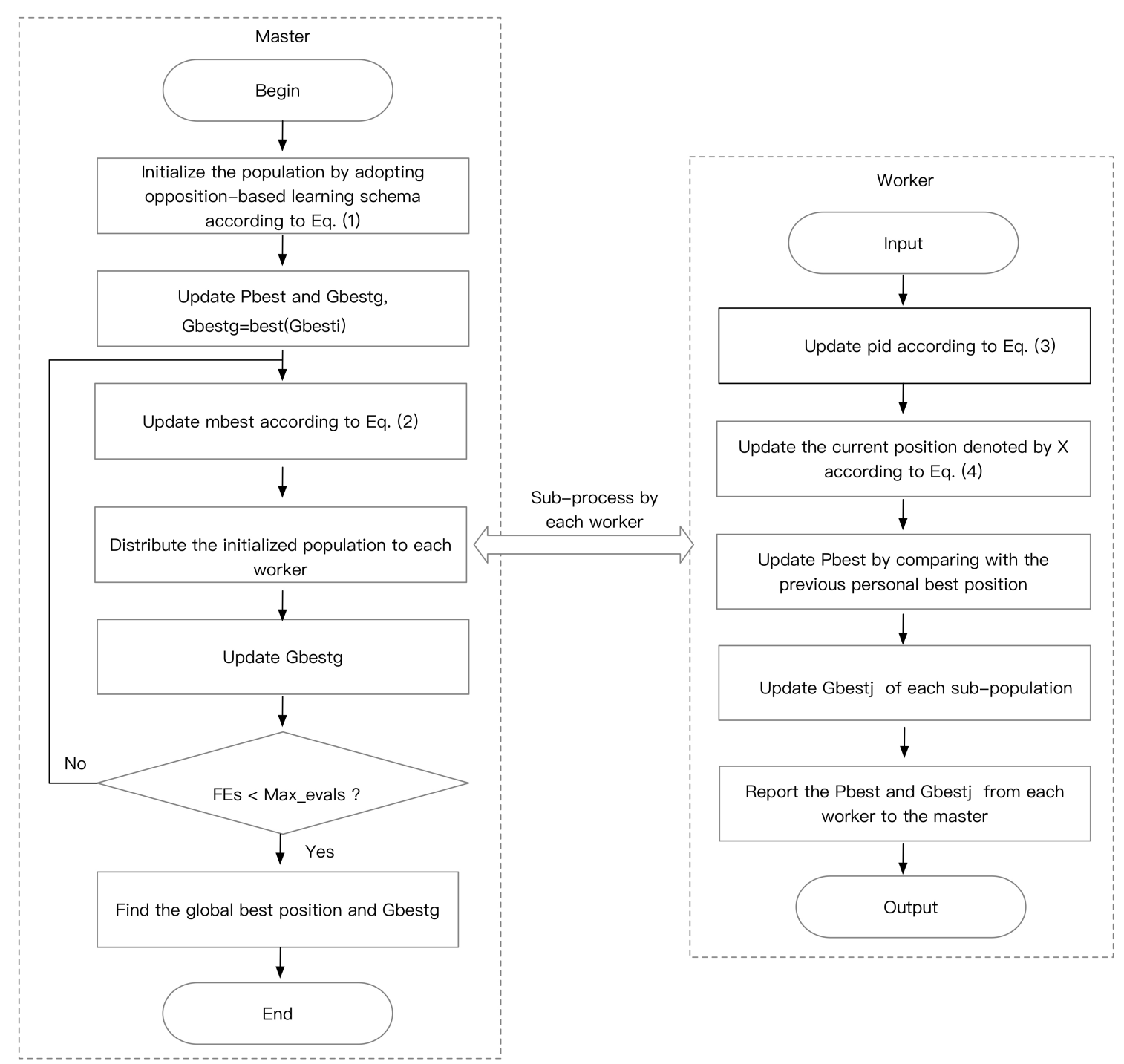

Figure 4. The evolution process of the distributed quantum-behaved particle swarm optimization (SDQPSO) algorithm.

The main steps of the SDQPSO algorithm are as follows:

1. The population is initialized by adopting opposition-based learning scheme on the master, where the population size is $M$, the current position is $X=\left(X_{1}, X_{2}, \cdots, X_{d}\right)$, the personal best position

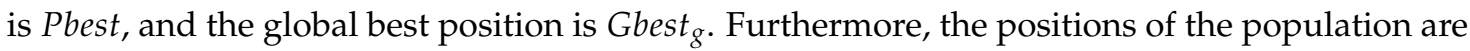
within the range of $\left[X_{\min }, X_{\max }\right]$.

2. According to Equation (2), the mean best position denoted by mbest is updated by averaging the personal best position on the master.

3. The population is divided into $P$ sub-populations on the master, then the sub-population and mbest are distributed to each worker. Moreover, the computing resource is distributed to each subpopulation, and the search space is partitioned into several search subspaces.

4. According to Equation (3), the local attractor of the particle denoted by $P_{i d}$ is updated on the worker, where $\mathrm{i}=1,2, \ldots \mathrm{M}$.

5. Each subpopulation on the worker evolves independently generation by generation, and the position of each particle is updated according to the Equation (4).

6. The fitness value of each particle is evaluated on the worker. When compared with Pbest, if the current fitness value is better than the previous personal best position, then Pbest is updated, otherwise not. 
7. For each worker, the particle with the best fitness value of each subpopulation is set as Gbest $t_{j}$.

8. The updated Gbest ${ }_{j}$ of each subpopulation and the current position are sent to the master.

9. The Gbest $t_{j}$ of all the sub-populations on the worker is sent to the master, where $j=1,2, \ldots P$. Furthermore, each Gbest $t_{j}$ is compared with Gbest $t_{g}$, if it is less than Gbest ${ }_{g}$, the global best position is updated, otherwise not. Then the updated Gbest $\mathrm{g}$ is distributed to each worker.

Repeat Step 2-Step 9. The evolution process is finished if the termination condition is reached, and finally the global best position denoted by Gbest $t_{g}$ and the personal best position denoted by Pbest are achieved; if the termination condition is not reached, go to Step 2.

\section{Results and Discussion}

\subsection{Experimental Environment and Parameter Settings}

\subsubsection{Experimental Environment}

The experimental environment of the Spark cluster is configured as follows: Dell PowerEdge R930 with Xeon(R) CPU E7-4820V4 @ 2.0 GHz *20, 256 GB memory and 6 TB hard disk. The configuration of the Spark platform is described in Table 1, where the distributed cluster environment is virtualized with VMware, and the Spark cluster consists of four nodes.

Table 1. Spark cluster for distributed computing.

\begin{tabular}{cc}
\hline Spark Cluster & Platform Configuration \\
\hline Distributed computing nodes & 1 Master and 3 Workers \\
System & VMware ESXi 6.5.0, Ubuntu 16.04 \\
Framework version & Hadoop 3.2.1, Spark 2.4.5 \\
Software version & Scala 2.11.12, java 1.8.0, Sbt 1.1.6 \\
\hline
\end{tabular}

\subsubsection{Test Problems and Parameter Settings}

For the large-scale optimization problem, two typical test functions with different time complexity were selected to verify the performance of SDQPSO. As shown in Table 2, the test functions represent five different types of optimization problems, and the time complexity of test functions is linear or quadratic. Furthermore, four test functions were selected with linear time complexity for low-cost optimization problems: Sphere, Rosenbrock, Rastrigin, Griewank; a test function with quadratic time complexity was selected for high-cost optimization problems: Schwefel 1.2. In addition, each experiment was run 30 times independently.

Table 2. Test functions.

\begin{tabular}{cc}
\hline Test Function & Name \\
\hline$F_{1}(x)=\sum_{i=1}^{n} x_{i}^{2}$ & Sphere \\
$F_{2}(x)=\sum_{i=1}^{n-1}\left[100\left(x_{i+1}-x_{i}^{2}\right)^{2}+\left(x_{i}-1\right)^{2}\right]$ & Rosenbrock \\
$F_{3}(x)=\sum_{i=1}^{n}\left[x_{i}^{2}-10 \cos \left(2 \pi x_{i}\right)+10\right]$ & Rastrigin \\
$F_{4}(x)=1 / 4000 \sum_{i=1}^{n} x_{i}^{2}-\prod_{i=1}^{n} \cos \left(x_{i} / \sqrt{i}\right)+1$ & Griewank \\
$F_{5}(x)=\sum_{i=1}^{n}\left(\sum_{j=1}^{i} x_{j}\right)^{2}$ & Schwefel 1.2 \\
\hline
\end{tabular}

SDQPSO is compared with three different existing distributed particle swarm optimizers; namely, Spark-based distributed particle swarm optimizer (SPSO) [37], Spark-based comprehensive learning particle swarm optimizer (SCLPSO) [38], and Spark-based aging leader and challengers particle swarm optimizer (SALCPSO) [39]. All the experimental parameters of the compared algorithms are set the same as the original paper. The population size is set to 100 , and $\beta$ is set to 0.5 in SDQPSO. In this 
paper, the performance of the SDQPSO algorithm based on the SDCEA framework is tested from three different perspectives: test functions with different time complexity, different dimensions, and different function evaluations, respectively. Based on 30 independent runs, optimization performance and computational cost are analyzed. For computational cost, the mean running time and function evaluation time are reported.

\subsection{Comparison with SDQPSO, SPSO, SCLPSO, and SALCPSO}

\subsubsection{Optimization Performance on Four Low-Cost Test Functions}

To evaluate the optimization performance of SDQPSO, SDQPSO is compared with SPSO, SCLPSO, and SALCPSO. Since Spark is a distributed framework that is efficient for the large-scale optimization problem, the test function is designed with a higher dimension and more expensive computational cost. The dimension is set to 100,000, and the function evaluations are set to 5000. For F1, F2, F3, and F4, the results obtained by SDQPSO, SPSO, SCLPSO, and SALCPSO are shown in Table 3. Furthermore, Wilcoxon's rank sum test is used to represent Robust Statistical Tests to demonstrate the performance of the proposed SDQPSO. The significance level is set to 0.05 . " + " means the compared algorithm performs significantly better than the proposed SDQPSO; "-" means the compared algorithm performs statistically worse than the proposed SDQPSO; " =" refers to non-comparable between the compared algorithm and the proposed SDQPSO.

In this paper, mean value, maximum value (max value), minimum value (min value), and standard variance are listed to evaluate the optimization performance, and the better-obtained values are indicated with bold fonts. As shown in Table 3, it can be found that the proposed SDQPSO has achieved better optimums on F2 in terms of the mean value, max value, min value, and standard variance. Furthermore, SALCPSO has achieved a better standard variance on F1, F3, and F4, whereas SDQPSO is better for mean value, max value, and min value. In addition, the optimization performance of the four compared algorithms has a relatively smaller difference on F3. In comparison with SPSO, SCLPSO, and SALCPSO, SDQPSO can achieve relatively competitive performance on F1, F2, and F4. According to the statistical results of 30 times on the functions, it can be concluded that the optimization performance of SDQPSO is the comprehensively best on all values, and SPSO is the second-best.

Table 3. Optimization performance on four functions.

\begin{tabular}{|c|c|c|c|c|c|}
\hline Function & & SDQPSO & SPSO & SCLPSO & SALCPSO \\
\hline \multirow{4}{*}{ F1 } & Min & $2.74 \times 10^{5}$ & $1.59 \times 10^{6}$ & $1.75 \times 10^{6}$ & $2.69 \times 10^{6}$ \\
\hline & Mean & $2.86 \times 10^{5}$ & $1.61 \times 10^{6}(-)$ & $1.75 \times 10^{6}(-)$ & $2.70 \times 10^{6}(-)$ \\
\hline & $\operatorname{Max}$ & $2.97 \times 10^{5}$ & $1.62 \times 10^{6}$ & $1.76 \times 10^{6}$ & $2.70 \times 10^{6}$ \\
\hline & Std & $7.41 \times 10^{3}$ & $9.97 \times 10^{3}$ & $6.63 \times 10^{3}$ & $4.33 \times 10^{3}$ \\
\hline \multirow{4}{*}{ F2 } & Min & $1.31 \times 10^{9}$ & $7.28 \times 10^{9}$ & $7.35 \times 10^{9}$ & $1.69 \times 10^{10}$ \\
\hline & Mean & $1.32 \times 10^{9}$ & $7.38 \times 10^{9}(-)$ & $7.44 \times 10^{9}(-)$ & $1.69 \times 10^{10}(-)$ \\
\hline & $\operatorname{Max}$ & $1.32 \times 10^{9}$ & $7.49 \times 10^{9}$ & $7.53 \times 10^{9}$ & $1.70 \times 10^{10}$ \\
\hline & Std & $3.44 \times 10^{6}$ & $6.74 \times 10^{7}$ & $5.30 \times 10^{7}$ & $3.26 \times 10^{7}$ \\
\hline \multirow{4}{*}{ F3 } & Min & $1.27 \times 10^{6}$ & $2.55 \times 10^{6}$ & $2.73 \times 10^{6}$ & $3.61 \times 10^{6}$ \\
\hline & Mean & $1.28 \times 10^{6}$ & $2.59 \times 10^{6}(-)$ & $2.74 \times 10^{6}(-)$ & $3.61 \times 10^{6}(-)$ \\
\hline & $\operatorname{Max}$ & $1.29 \times 10^{6}$ & $2.60 \times 10^{6}$ & $2.76 \times 10^{6}$ & $3.62 \times 10^{6}$ \\
\hline & Std & $7.03 \times 10^{3}$ & $1.55 \times 10^{4}$ & $7.81 \times 10^{3}$ & $3.37 \times 10^{3}$ \\
\hline \multirow{4}{*}{ F4 } & Min & $6.73 \times 10^{1}$ & $3.97 \times 10^{2}$ & $4.36 \times 10^{2}$ & $6.73 \times 10^{2}$ \\
\hline & Mean & $7.16 \times 10^{1}$ & $4.03 \times 10^{2}(-)$ & $4.37 \times 10^{2}(-)$ & $6.75 \times 10^{2}(-)$ \\
\hline & $\operatorname{Max}$ & $7.34 \times 10^{1}$ & $4.10 \times 10^{2}$ & $4.40 \times 10^{2}$ & $6.76 \times 10^{2}$ \\
\hline & Std & $1.93 \times 10^{0}$ & $3.43 \times 10^{0}$ & $1.38 \times 10^{0}$ & $8.46 \times 10^{-1}$ \\
\hline$+/=/-$ & & & $0 / 0 / 4$ & $0 / 0 / 4$ & $0 / 0 / 4$ \\
\hline
\end{tabular}




\subsubsection{Optimization Performance under Different Function Dimensions}

To test the performance of the SDQPSO under different function dimensions, the Schwefel 1.2 function with quadratic time complexity is selected since it can highlight the advantage of the distributed computing using Spark. For Schwefel 1.2, the dimension is set to 10, 100, 1000, 10,000, 100,000 . Furthermore, based on our experience, the computational cost of the Schwefel 1.2 function is very high for large-scale optimization problems, so the function evaluation is set to 500. In addition, since the optimization performance of the distributed optimizer under different dimensions is a crucial issue, the comparison of different function evaluations is specifically described in Section 5.2.3 when the function dimension is set to 100,000 .

The optimization performance of SDQPSO, SPSO, SCLPSO, and SALCPSO under different function dimensions is shown in Table 4 . It can be seen from Table 4 that SDQPSO has achieved better optimums on the mean value, max value, min value, and standard variance, except that SPSO has achieved the best standard variance when the dimension is 100,000 . From Table 4 , it is clear that the optimum obtained by SDQPSO is much closer to the optimum in theory when the dimension is 10. In addition, the difficulty of obtaining the optimal solution increases gradually with the dimension increasing. Furthermore, the performance of SCLPSO is relatively worse than the other three algorithms, but SDQPSO, SPSO, SCLPSO, and SALCPSO can achieve a relatively close optimum. Comprehensively, on the four values, SPSO is the second-best, and then SALCPSO, and the proposed SDQPSO adopting the opposition-based learning scheme has a competitive optimization performance.

Table 4. Optimization performance under different function dimensions.

\begin{tabular}{|c|c|c|c|c|c|}
\hline Dimension & & SDQPSO & SPSO & SCLPSO & SALCPSO \\
\hline \multirow{4}{*}{10} & Min & $1.22 \times 10^{0}$ & $7.19 \times 10^{0}$ & $3.29 \times 10^{1}$ & $2.19 \times 10^{1}$ \\
\hline & Mean & $6.94 \times 10^{0}$ & $1.81 \times 10^{1}(-)$ & $9.93 \times 10^{1}(-)$ & $4.43 \times 10^{1}(-)$ \\
\hline & $\operatorname{Max}$ & $1.99 \times 10^{1}$ & $2.69 \times 10^{1}$ & $1.60 \times 10^{2}$ & $9.17 \times 10^{1}$ \\
\hline & Std & $4.97 \times 10^{0}$ & $5.58 \times 10^{0}$ & $2.98 \times 10^{1}$ & $1.56 \times 10^{1}$ \\
\hline \multirow{4}{*}{100} & Min & $1.21 \times 10^{3}$ & $1.79 \times 10^{3}$ & $6.41 \times 10^{3}$ & $3.47 \times 10^{3}$ \\
\hline & Mean & $2.00 \times 10^{3}$ & $2.96 \times 10^{3}(-)$ & $9.00 \times 10^{3}(-)$ & $5.64 \times 10^{3}(-)$ \\
\hline & Max & $2.60 \times 10^{3}$ & $4.20 \times 10^{3}$ & $1.21 \times 10^{4}$ & $1.01 \times 10^{4}$ \\
\hline & Std & $4.35 \times 10^{2}$ & $5.63 \times 10^{2}$ & $1.46 \times 10^{3}$ & $1.32 \times 10^{3}$ \\
\hline \multirow{4}{*}{1000} & Min & $1.47 \times 10^{5}$ & $1.51 \times 10^{5}$ & $5.23 \times 10^{5}$ & $2.79 \times 10^{5}$ \\
\hline & Mean & $2.34 \times 10^{5}$ & $2.85 \times 10^{5}(-)$ & $8.73 \times 10^{5}(-)$ & $5.24 \times 10^{5}(-)$ \\
\hline & Max & $2.94 \times 10^{5}$ & $3.94 \times 10^{5}$ & $1.25 \times 10^{6}$ & $8.39 \times 10^{5}$ \\
\hline & Std & $4.15 \times 10^{4}$ & $5.28 \times 10^{4}$ & $1.95 \times 10^{5}$ & $1.17 \times 10^{5}$ \\
\hline \multirow{4}{*}{10,000} & Min & $1.50 \times 10^{7}$ & $1.89 \times 10^{7}$ & $5.44 \times 10^{7}$ & $3.85 \times 10^{7}$ \\
\hline & Mean & $2.53 \times 10^{7}$ & $2.98 \times 10^{7}(-)$ & $8.98 \times 10^{7}(-)$ & $5.44 \times 10^{7}(-)$ \\
\hline & $\operatorname{Max}$ & $3.56 \times 10^{7}$ & $4.68 \times 10^{7}$ & $1.53 \times 10^{8}$ & $7.93 \times 10^{7}$ \\
\hline & Std & $5.68 \times 10^{6}$ & $6.23 \times 10^{6}$ & $1.95 \times 10^{7}$ & $1.01 \times 10^{7}$ \\
\hline \multirow{4}{*}{100,000} & Min & $1.75 \times 10^{9}$ & $2.26 \times 10^{9}$ & $4.44 \times 10^{9}$ & $3.25 \times 10^{9}$ \\
\hline & Mean & $2.59 \times 10^{9}$ & $3.16 \times 10^{9}(-)$ & $8.89 \times 10^{9}(-)$ & $5.47 \times 10^{9}(-)$ \\
\hline & Max & $3.42 \times 10^{9}$ & $4.18 \times 10^{9}$ & $1.35 \times 10^{10}$ & $9.05 \times 10^{9}$ \\
\hline & Std & $7.16 \times 10^{8}$ & $5.26 \times 10^{8}$ & $2.11 \times 10^{9}$ & $1.32 \times 10^{9}$ \\
\hline$+/=/-$ & & & $0 / 0 / 5$ & $0 / 0 / 5$ & $0 / 0 / 5$ \\
\hline
\end{tabular}

\subsubsection{Optimization Performance under Different Function Evaluations}

For the large-scale optimization problem, the optimization performance of SDQPSO under different function evaluations is discussed. A high-cost Schwefel 1.2 function is selected. Moreover, five function evaluations are set to 1000, 2000, 3000, 4000, 5000, and the dimension is set to 100,000.

The optimization performance of SDQPSO, SPSO, SCLPSO, and SALCPSO under different function evaluations are shown in Table 5. From Table 5, it can be found that SDQPSO and SPSO 
have better optimums. When the function evaluation is set to 1000, SDQPSO could obtain the best optimum on min value, max value, and standard variance, but is worse than SPSO on the mean value. When the function evaluations are 2000 and 4000, SDQPSO could achieve a better standard variance but with a slightly inferior performance than SPSO on the optimum. Furthermore, when the function evaluations are 3000 and 5000, SDQPSO is very close to SPSO on the four values: mean value, max value, min value, and standard variance. The optimum obtained by SDQPSO has a superior performance when compared with SCLPSO and SALCPSO. In conclusion, SDQPSO could obtain a competitive performance for the large-scale and high-cost optimization problem.

Table 5. Optimization performance under different function evaluations.

\begin{tabular}{|c|c|c|c|c|c|}
\hline Evaluation & & SDQPSO & SPSO & SCLPSO & SALCPSO \\
\hline \multirow{4}{*}{1000} & Min & $1.43 \times 10^{9}$ & $1.49 \times 10^{9}$ & $4.20 \times 10^{9}$ & $2.61 \times 10^{9}$ \\
\hline & Mean & $2.54 \times 10^{9}$ & $2.21 \times 10^{9}(=)$ & $7.96 \times 10^{9}(-)$ & $4.20 \times 10^{9}(-)$ \\
\hline & $\operatorname{Max}$ & $2.99 \times 10^{9}$ & $3.06 \times 10^{9}$ & $1.08 \times 10^{10}$ & $5.76 \times 10^{9}$ \\
\hline & Std & $4.10 \times 10^{8}$ & $4.48 \times 10^{8}$ & $1.59 \times 10^{9}$ & $6.80 \times 10^{8}$ \\
\hline \multirow{4}{*}{2000} & Min & $1.42 \times 10^{9}$ & $1.07 \times 10^{9}$ & $3.67 \times 10^{9}$ & $2.21 \times 10^{9}$ \\
\hline & Mean & $2.31 \times 10^{9}$ & $1.78 \times 10^{9}(+)$ & $6.82 \times 10^{9}(-)$ & $3.33 \times 10^{9}(-)$ \\
\hline & $\operatorname{Max}$ & $2.87 \times 10^{9}$ & $2.50 \times 10^{9}$ & $9.90 \times 10^{9}$ & $4.52 \times 10^{9}$ \\
\hline & Std & $3.21 \times 10^{8}$ & $3.40 \times 10^{8}$ & $1.50 \times 10^{9}$ & $6.74 \times 10^{8}$ \\
\hline \multirow{4}{*}{3000} & Min & $1.61 \times 10^{9}$ & $1.13 \times 10^{9}$ & $3.76 \times 10^{9}$ & $2.17 \times 10^{9}$ \\
\hline & Mean & $2.32 \times 10^{9}$ & $1.64 \times 10^{9}(+)$ & $5.59 \times 10^{9}(-)$ & $3.20 \times 10^{9}(-)$ \\
\hline & $\operatorname{Max}$ & $2.75 \times 10^{9}$ & $2.54 \times 10^{9}$ & $8.09 \times 10^{9}$ & $3.92 \times 10^{9}$ \\
\hline & Std & $4.12 \times 10^{8}$ & $3.01 \times 10^{8}$ & $1.05 \times 10^{9}$ & $4.58 \times 10^{8}$ \\
\hline \multirow{4}{*}{4000} & Min & $1.88 \times 10^{9}$ & $1.10 \times 10^{9}$ & $3.57 \times 10^{9}$ & $2.20 \times 10^{9}$ \\
\hline & Mean & $2.16 \times 10^{9}$ & $1.51 \times 10^{9}(+)$ & $5.67 \times 10^{9}(-)$ & $2.89 \times 10^{9}(-)$ \\
\hline & Max & $2.44 \times 10^{9}$ & $2.02 \times 10^{9}$ & $7.52 \times 10^{9}$ & $4.08 \times 10^{9}$ \\
\hline & Std & $1.85 \times 10^{8}$ & $2.04 \times 10^{8}$ & $9.85 \times 10^{8}$ & $4.50 \times 10^{8}$ \\
\hline \multirow{4}{*}{5000} & Min & $1.63 \times 10^{9}$ & $1.16 \times 10^{9}$ & $4.11 \times 10^{9}$ & $2.02 \times 10^{9}$ \\
\hline & Mean & $2.11 \times 10^{9}$ & $1.53 \times 10^{9}(+)$ & $5.52 \times 10^{9}(-)$ & $2.68 \times 10^{9}(-)$ \\
\hline & $\operatorname{Max}$ & $2.85 \times 10^{9}$ & $1.96 \times 10^{9}$ & $7.50 \times 10^{9}$ & $4.01 \times 10^{9}$ \\
\hline & Std & $3.80 \times 10^{8}$ & $1.93 \times 10^{8}$ & $8.23 \times 10^{8}$ & $4.14 \times 10^{8}$ \\
\hline$+/=/-$ & & & $4 / 1 / 0$ & $0 / 0 / 5$ & $0 / 0 / 5$ \\
\hline
\end{tabular}

\subsection{Performance on the Running Time and Function Evaluation Proportion}

\subsubsection{Running Time and Function Evaluation Proportion on Four Low-Cost Test Functions}

For the time metrics, the running time and function evaluation time were reported. The function dimension is set to 100,000 , and the function evaluation is set to 5000. The time performance of SDQPSO, SPSO, SCLPSO, and SALCPSO on four different functions is shown in Figure 5, where Figure 5a-d represent the time metrics of Sphere, Rosenbrock, Rastrigin, and Griewank, respectively. For Figure 5, different colored histograms represent different time metrics, blue for the running time, and orange for the function evaluation time.

For the running time, it can be found that SCLPSO has the highest computational cost on the four low-cost test functions with linear time complexity, followed by SDQPSO. Furthermore, SPSO and SALCPSO could obtain lower computational cost, and the running time of the two algorithms are very close. Furthermore, the function evaluation time of SDQPSO, SPSO, SCLPSO, and SALCPSO is very close, and the function evaluation proportion is small which means that the function evaluation only takes less time. Generally speaking, compared with SPSO, SCLPSO, and SALCPSO, SDQPSO can keep a relatively competitive performance on the time metric. 


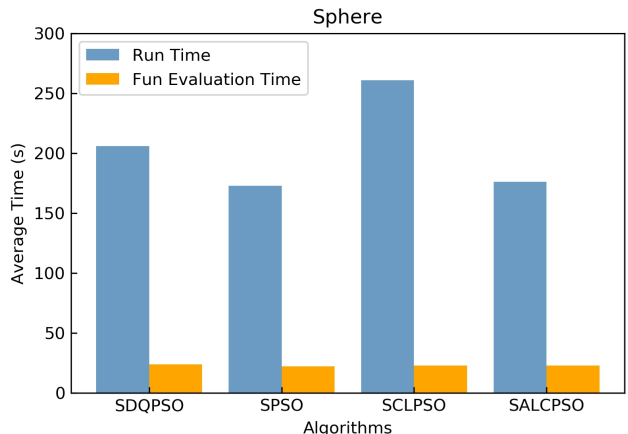

( a )

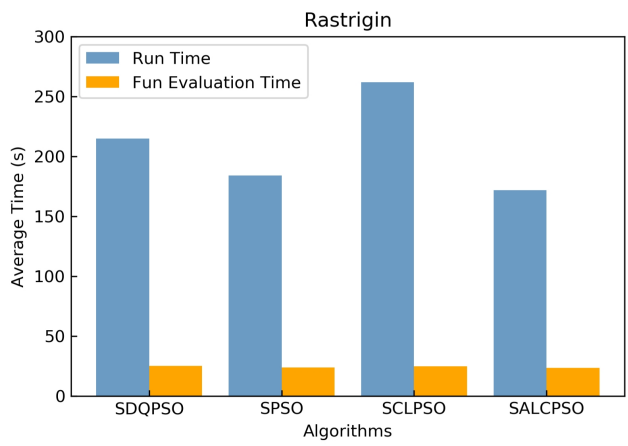

(c)

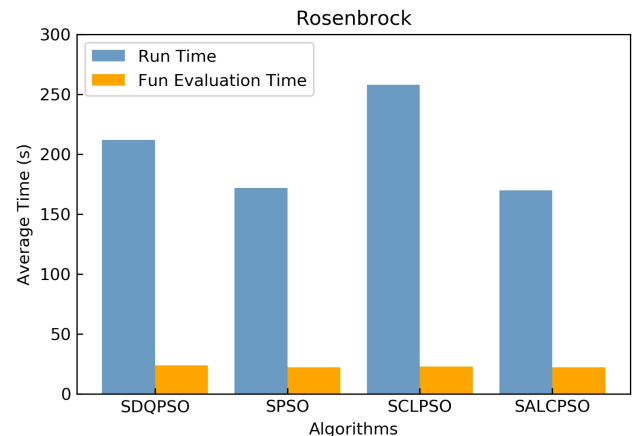

( b )

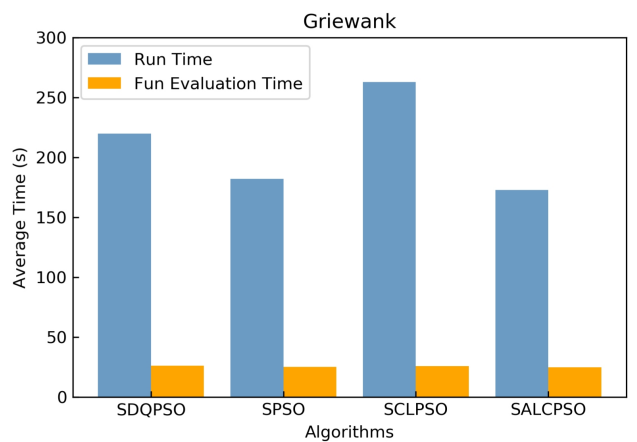

(d)

Figure 5. The time metrics on Sphere, Rosenbrock, Rastrigin, Griewank. (a) Time on Sphere, (b) time on Rosenbrock, (c) time on Rastrigin, (d) time on Griewank.

The function evaluation proportion represents the proportion between the function evaluation time and the running time. The function evaluation proportion of Sphere, Rosenbrock, Rastrigin, and Griewank is shown in Figure 6, where four different colors represent the evaluation proportion of different functions, respectively. From Figure 6, it can be found that the function evaluation proportion of SCLPSO is the best, followed by SDQPSO, SPSO, and SALCPSO. However, the result shows that the function evaluation is less time-consuming on the four low-cost test functions, ranging from $7.5 \%$ to $15 \%$. In summary, the experimental results verified the time complexity of the four low-cost test functions. Furthermore, the SDQPSO using the opposition-based learning scheme could obtain better function evaluation proportion when faced with the low-cost optimization problem.

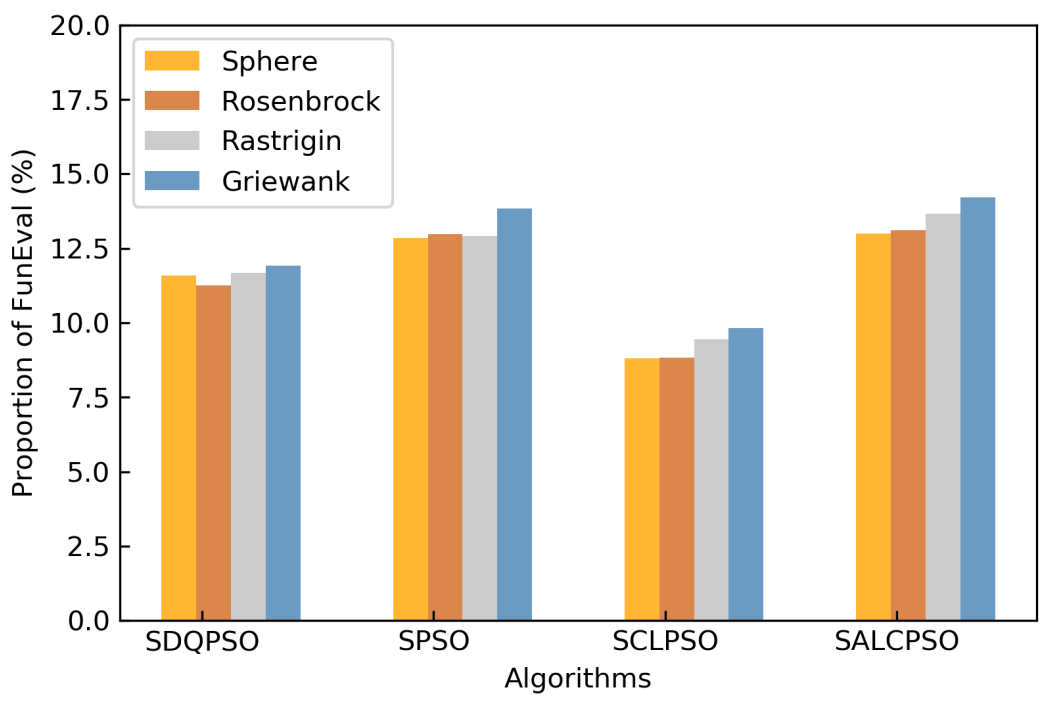

Figure 6. The fun evaluation proportion on Sphere, Rosenbrock, Rastrigin, and Griewank. 


\subsubsection{Running Time and Function Evaluation Proportion under Different Dimensions}

For the large-scale optimization problem, a high-cost Schwefel 1.2 function with quadratic time complexity is tested. The dimensions are set to $10,100,1000,10,000,100,000$. The mean running times of SDQPSO, SPSO, SCLPSO, and SALCPSO under different dimensions are shown in Figure 7, it is clear that the mean running time of the four algorithms is very close. When the dimension is less than 1000, the mean running time of SDQPSO and SPSO, SCLPSO, and SALCPSO is less than 1 second, and when the dimension is 10,000, the mean running time increases to nearly 10 seconds. In addition, the mean running time increases drastically to more than 100 seconds when the dimension is set to 100,000 .

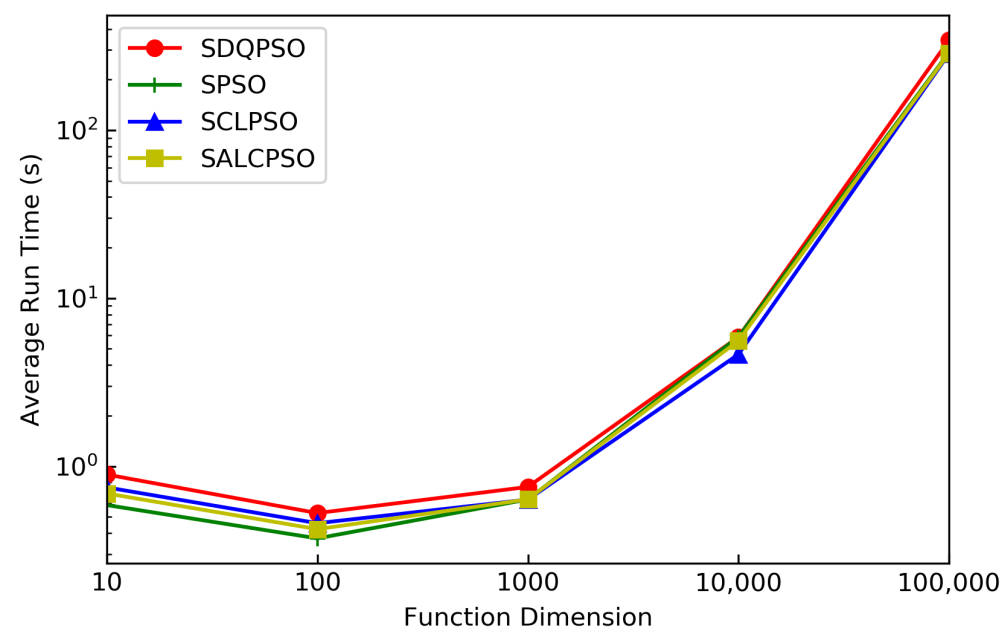

Figure 7. The running time under different dimensions.

The function evaluation proportion of SDQPSO, SPSO, SCLPSO, and SALCPSO under different dimensions are shown in Figure 8. Since the computational cost of the algorithm is low, which results in the function evaluation proportion being relatively high when dimensions are set to 10 and 100 . When the dimension is 1000 , the function evaluation proportion of all algorithms is lower than that of other dimensions, dimension and the function evaluation proportion of the four algorithms increases with the further increase of dimensions. Furthermore, SCLPSO has the largest function evaluation proportion among all algorithms, reaching nearly $75 \%$ when the dimension is 1000, and SDQPSO outperforms SCLPSO.

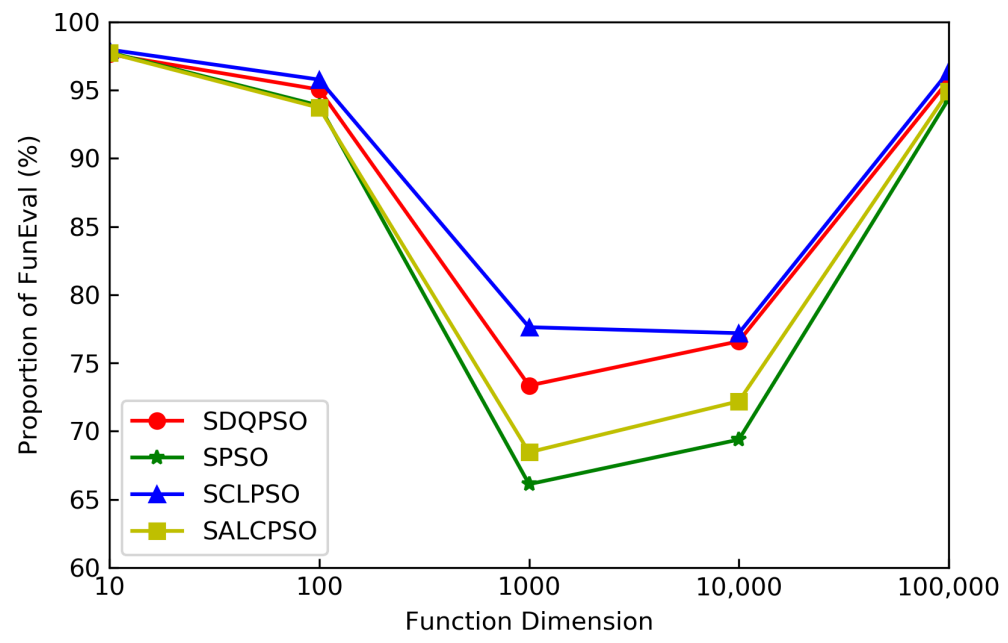

Figure 8. The function evaluation proportion under different dimensions. 


\subsubsection{Running Time and Function Evaluation Proportion under Different Function Evaluations}

To verify the performance of SDQPSO under different function evaluations, the function evaluations are set to 1000, 2000, 3000, 4000, 5000, respectively. In addition, the dimension is set to 100,000 , and the high-cost Schwefel 1.2 function is selected for the test. The mean running time of SDQPSO, SPSO, SCLPSO, and SALCPSO under different function evaluations is shown in Figure 9. From Figure 9, it can be found that the running time of the four algorithms increases linearly with the function evaluation increasing. Furthermore, the running time of SDQPSO, SPSO, SCLPSO, and SALCPSO is in an order of magnitude, and is very close to each other.

The function evaluation proportion under different function evaluations is illustrated in Figure 10. In comparison with SPSO, SALCPSO, and SDQPSO, the decline of function evaluation proportion of SCLPSO is the relatively best with the function evaluations increasing. Furthermore, for the function evaluation proportion, the proposed SDQPSO has achieved the second-best while SCLPSO is the best among all algorithms when the dimension ranges from 2000 to 5000 . Further analysis is given in Figure 10, it can be seen that the SDQPSO, SPSO, SCLPSO, and SALCPSO have a higher function evaluation proportion for the large-scale and high-cost optimization problem. Generally speaking, the experimental result verified the characteristics of the Schwefel 1.2 function, which means that the computational cost of the function with quadratic time complexity is high.

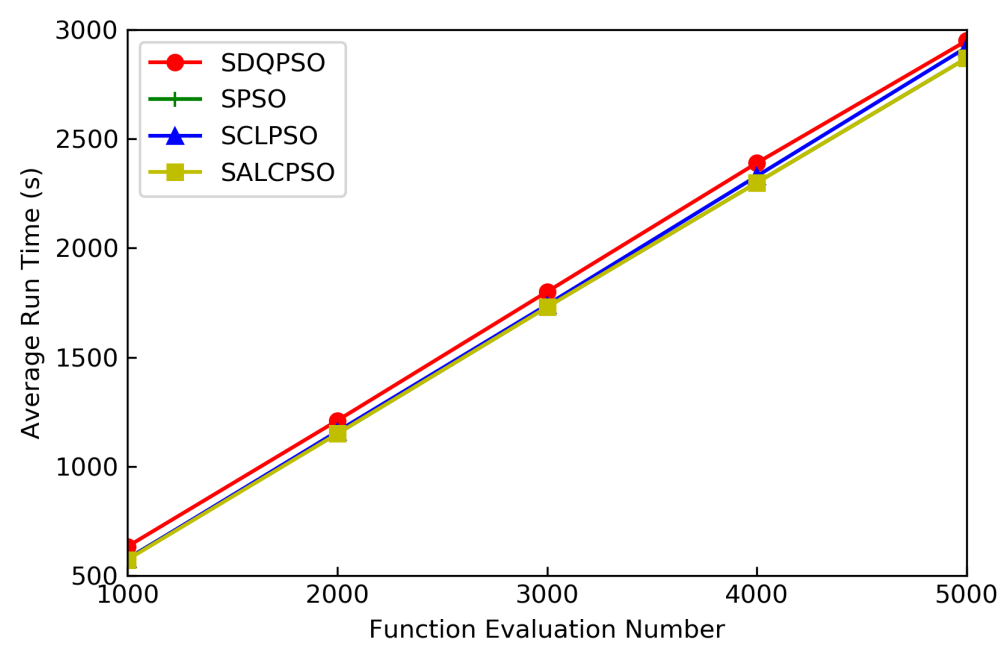

Figure 9. The running time under different function evaluations.

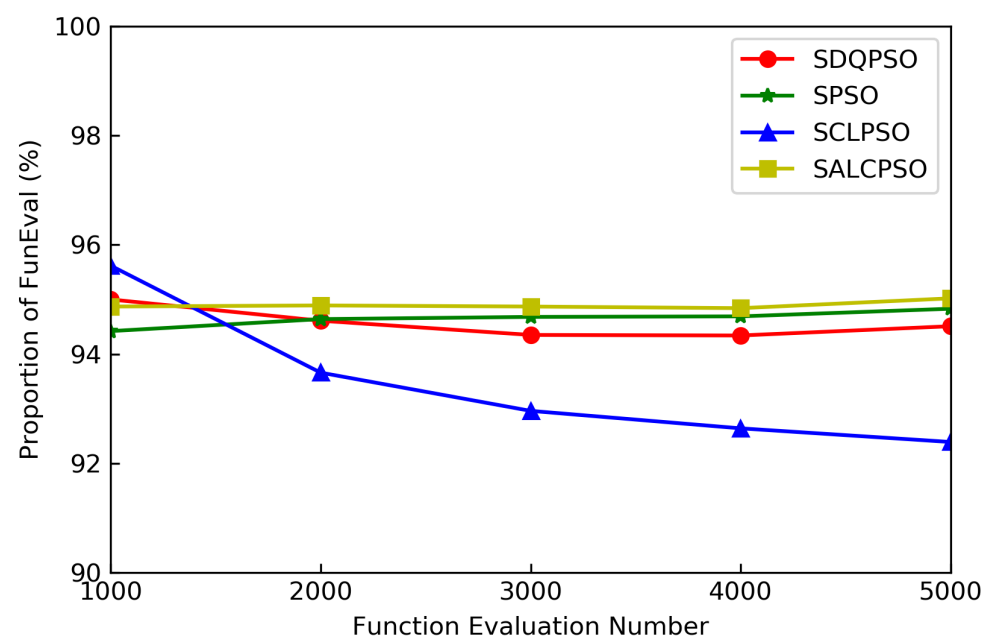

Figure 10. The function evaluation proportion under different function evaluations. 


\subsection{Discussion}

With the ongoing rapid accumulation of data scale, and the increasing complexity of real-world optimization problems, new challenges faced by traditional intelligent optimization algorithms are increasing. To deal with the massive, high-dimensional, and dynamic challenge faced by the large-scale optimization problem, a distributed cooperative evolutionary algorithm framework using Spark is proposed first. Furthermore, the proposed framework can be extended with the increasing computing resources, e.g., it can be extended to 10 nodes, 100 nodes. It means that the SDCEA is very flexible and has good scalability regardless of the node size.

More importantly, the distributed optimization algorithm based on the SDCEA can be applied to address the challenge in the other similar optimization problems. That is, the crucial issue is how to implement an algorithm in a distributed way, so further study is deserved focusing on the distributed optimization framework. When faced with the problem of lower computational efficiency and higher computational cost caused by big data, the SDCEA proposed in this paper and the distributed optimization algorithms can be applied to solve the above-mentioned challenges. Therefore, how to design the distributed and cooperative co-evolution from the serial algorithm to the big data-driven optimization is significant for real-world optimization problems.

Through further analysis of the results, it can be found that for the large-scale and low-cost optimization problem, the performance on time metrics of the SDCEA is not competitive since communication takes a long time through distributed computing. This means that distributed computing is an effective strategy when faced with big data, and the larger the data are, the greater the advantage will be. The running time of the algorithm mainly depends on the parallelism of the algorithm, and the computational cost of each subpopulation directly affects the convergence speed of the whole population. Too much parallelism means that there are too many tasks, which can increase the overall computational cost. Furthermore, too few partitions can lead to unreasonable use of computing resources, which increases the memory requirements for each worker, and if the partition is not reasonable, which can result in data skew problems. Therefore, how to divide data in parallel according to computing resources and search space is of great significance.

The communication of the master-slave distribution model is very time-consuming and inefficient; thus, how to carry out the cooperation to reduce the communication time among the individuals of each subpopulation is worthy of further discussion. Furthermore, how to design and implement a novel and efficient framework based on big data platforms, e.g., MapReduce and Spark, to meet the increasing requirements when faced with big data is still significant for large-scale optimization problems.

\section{Conclusions}

In order to address the challenge faced by the big data-driven optimization problem, this paper first provides a literature review of the distributed intelligent optimization algorithms. Second, a distributed cooperative evolutionary algorithm framework using Spark is proposed, which combines distributed computing, population distribution, data distribution, and distributed co-evolution. Third, the SDQPSO by adopting the opposition-based learning scheme to initialize and be implemented in parallel based on the SDCEA framework. Finally, the performance of the SDQPSO under different functions, different dimensions, and different fitness evaluations are discussed, and three distributed optimizers, namely, SPSO, SCLPSO, and SALCPSO are selected to compare.

For the large-scale optimization problem, the proposed SDQPSO can obtain relatively better optimum values on low-cost functions. Moreover, for the high-cost and large-scale optimization problem, it could obtain a relatively competitive performance on function evaluation proportion and computational cost. Furthermore, the results show that the opposition-based learning scheme improves the probability of the population to obtain a better search space without prior knowledge. In conclusion, the proposed SDCEA framework can improve search efficiency by using Spark, and it has high scalability that can be applied to address the complexity of large-scale optimization problems. 
Author Contributions: Conceptualization, Z.Z.; methodology, W.W.; validation, G.P.; formal analysis, Z.Z. and G.P.; investigation, Z.Z.; resources, Z.Z.; data curation, G.P.; writing-original draft preparation, Z.Z.; writing-review and editing, W.W.; visualization, G.P.; supervision, W.W.; project administration, W.W.; funding acquisition, W.W. All authors have read and agreed to the published version of the manuscript.

Funding: This work was funded by the National Natural Science Foundation of China grant numbers 61873240. The funding body did not play any role in the design of the study and collection, analysis, and interpretation of data and writing the manuscript.

Acknowledgments: The authors wish to thank the editors and anonymous reviewers for their valuable comments and helpful suggestions which greatly improved the paper's quality. We would like to thank Marilyn E. Gartley for excellent and professional copy-editing of the paper. This work was cooperated with the Department of Computer Science and Engineering at University of South Carolina.

Conflicts of Interest: The authors declare no conflict of interest.

\section{References}

1. Gong, Y.J.; Chen, W.N.; Zhan, Z.H.; Zhang, J.; Li, Y.; Zhang, Q.; Li, J.J. Distributed evolutionary algorithms and their models: A survey of the state-of-the-art. Appl. Soft Comput. 2015, 34, 286-300. [CrossRef]

2. Wang, W.L. Artificial Intelligence: Principles and Applications; Higher Education Press: BeiJing, China, 2020.

3. Wang, W.L.; Zhang, Z.J.; Gao, N.; Zhao, Y.W. Research progress of big data analytics methods based on artificial intelligence technology. Comput. Integr. Manuf. Syst. 2019, 25, 529-547.

4. Dean, J.; Ghemawat, S. MapReduce: Simplified Data Processing on Large Clusters. Commun. ACM 2008, 51, 107-113.

5. McNabb, A.W.; Monson, C.K.; Seppi, K.D. Parallel pso using mapreduce. In Proceedings of the 2007 IEEE Congress on Evolutionary Computation, Singapore, 25-28 September 2007; pp. 7-14.

6. Zaharia, M.; Chowdhury, M.; Franklin, M.J.; Shenker, S.; Stoica, I. Spark: Cluster computing with working sets. HotCloud 2010, 10, 95.

7. Ghasemi, P.; Khalili-Damghani, K.; Hafezalkotob, A.; Raissi, S. Uncertain multi-objective multi-commodity multi-period multi-vehicle location-allocation model for earthquake evacuation planning. Appl. Math. Comput. 2019, 350, 105-132. [CrossRef]

8. Ghasemi, P.; Khalili-Damghani, K. A robust simulation-optimization approach for pre-disaster multi-period location-allocation-inventory planning. Math. Comput. Simul. 2020, 179, 69-95. [CrossRef]

9. Fakhrzad, M.B.; Goodarzian, F. A fuzzy multi-objective programming approach to develop a green closed-loop supply chain network design problem under uncertainty: Modifications of imperialist competitive algorithm. RAIRO-Oper. Res. 2019, 53, 963-990. [CrossRef]

10. Goodarzian, F.; Hosseini-Nasab, H.; Muñuzuri, J.; Fakhrzad, M.B. A multi-objective pharmaceutical supply chain network based on a robust fuzzy model: A comparison of meta-heuristics. Appl. Soft Comput. 2020, 92, 106331. [CrossRef]

11. Verdejo, H.; Pino, V.; Kliemann, W.; Becker, C.; Delpiano, J. Implementation of Particle Swarm Optimization (PSO) Algorithm for Tuning of Power System Stabilizers in Multimachine Electric Power Systems. Energies 2020, 13, 2093. [CrossRef]

12. Zhang, X.; Zou, D.; Shen, X. A novel simple particle swarm optimization algorithm for global optimization. Mathematics 2018, 6, 287. [CrossRef]

13. Yildizdan, G.; Baykan, O.K. A new hybrid BA-ABC algorithm for global optimization problems. Mathematics 2020, 8, 1749. [CrossRef]

14. Wei, C.L.; Wang, G.G. Hybrid Annealing Krill Herd and Quantum-Behaved Particle Swarm Optimization. Mathematics 2020, 8, 1403. [CrossRef]

15. Jin, C.; Vecchiola, C.; Buyya, R. MRPGA: An extension of MapReduce for parallelizing genetic algorithms. In Proceedings of the 2008 IEEE Fourth International Conference on eScience, Indianapolis, IN, USA, 7-12 December 2008; pp. 214-221.

16. Wu, H.; Ni, Z.W.; Wang, H.Y. MapReduce-based ant colony optimization. Comput. Integr. Manuf. Syst. 2012, $18,1503-1509$.

17. Cheng, X.; Xiao, N. Parallel implementation of dynamic positive and negative feedback ACO with iterative MapReduce model. J. Inf. Comput. Sci. 2013, 10, 2359-2370. [CrossRef] 
18. Xu, X.; Ji, Z.; Yuan, F.; Liu, X. A novel parallel approach of cuckoo search using MapReduce. In Proceedings of the 2014 International Conference on Computer, Communications and Information Technology (CCIT 2014), Beijing, China, 16-17 January 2014.

19. Al-Madi, N.; Aljarah, I.; Ludwig, S.A. Parallel glowworm swarm optimization clustering algorithm based on MapReduce. In Proceedings of the 2014 IEEE Symposium on Swarm Intelligence, Orlando, FL, USA, 9-12 December 2014; pp. 1-8.

20. Ding, W.P.; Lin, C.T.; Prasad, M.; Chen, S.B.; Guan, Z.J. Attribute equilibrium dominance reduction accelerator (DCCAEDR) based on distributed coevolutionary cloud and its application in medical records. IEEE Trans. Syst. Man Cybern. Syst. 2015, 46, 384-400. [CrossRef]

21. Hossain, M.S.; Moniruzzaman, M.; Muhammad, G.; Ghoneim, A.; Alamri, A. Big data-driven service composition using parallel clustered particle swarm optimization in mobile environment. IEEE Trans. Serv. Comput. 2016, 9, 806-817. [CrossRef]

22. Wang, Y.; Li, Y.; Chen, Z.; Xue, Y. Cooperative particle swarm optimization using MapReduce. Soft Comput. 2017, 21, 6593-6603. [CrossRef]

23. Li, Y.; Chen, Z.; Wang, Y.; Jiao, L.; Xue, Y. A novel distributed quantum-behaved particle swarm optimization. J. Optim. 2017, 2017. [CrossRef]

24. Ding, W.; Lin, C.T.; Chen, S.; Zhang, X.; Hu, B. Multiagent-consensus-MapReduce-based attribute reduction using co-evolutionary quantum PSO for big data applications. Neurocomputing 2018, 272, 136-153. [CrossRef]

25. Khalil, Y.; Alshayeji, M.; Ahmad, I. Distributed whale optimization algorithm based on MapReduce. Concurr. Comput. Pract. Exp. 2019, 31, e4872. [CrossRef]

26. Cao, B.; Li, W.; Zhao, J.; Yang, S.; Kang, X.; Ling, Y.; Lv, Z. Spark-based parallel cooperative co-evolution particle swarm optimization algorithm. In Proceedings of the 2016 IEEE International Conference on Web Services (ICWS), San Francisco, CA, USA, 27 June-2 July 2016; pp. 570-577.

27. Qi, R.Z.; Wang, Z.J.; Li, S.Y. A parallel genetic algorithm based on spark for pairwise test suite generation. J. Comput. Sci. Technol. 2016, 31, 417-427. [CrossRef]

28. Liu, P.; Ye, S.; Wang, C.; Zhu, Z. Spark-Based Parallel Genetic Algorithm for Simulating a Solution of Optimal Deployment of an Underwater Sensor Network. Sensors 2019, 19, 2717. [CrossRef] [PubMed]

29. Yuan, J. An Anomaly Data Mining Method for Mass Sensor Networks Using Improved PSO Algorithm Based on Spark Parallel Framework. J. Grid Comput. 2020, 18, 251-261. [CrossRef]

30. Peng, H.; Tan, X.; Deng, C.; Peng, S. SparkCUDE: A spark-based differential evolution for large-scale global optimisation. Int. J. High Perform. Syst. Archit. 2017, 7, 211-222. [CrossRef]

31. Teijeiro, D.; Pardo, X.C.; González, P.; Banga, J.R.; Doallo, R. Implementing parallel differential evolution on Spark. In Proceedings of the European Conference on the Applications of Evolutionary Computation, Porto, Portugal, 30 March-1 April 2016; pp. 75-90.

32. Sun, L.; Lin, L.; Li, H.; Gen, M. Large scale flexible scheduling optimization by a distributed evolutionary algorithm. Comput. Ind. Eng. 2019, 128, 894-904. [CrossRef]

33. Zaharia, M.; Chowdhury, M.; Das, T.; Dave, A.; Ma, J.; McCauly, M.; Franklin, M.J.; Shenker, S.; Stoica, I. Resilient distributed datasets: A fault-tolerant abstraction for in-memory cluster computing. In Proceedings of the 9th \{USENIX\} Symposium on Networked Systems Design and Implementation (\{NSDI\} 12), San Jose, CA, USA, 25-27 April 2012; pp. 15-28.

34. Barba-González, C.; García-Nieto, J.; Nebro, A.J.; Cordero, J.A.; Durillo, J.J.; Navas-Delgado, I.; Aldana-Montes, J.F. jMetalSP: A framework for dynamic multi-objective big data optimization. Appl. Soft Comput. 2018, 69, 737-748. [CrossRef]

35. Duan, Q.; Sun, L.; Shi, Y. Spark clustering computing platform based parallel particle swarm optimizers for computationally expensive global optimization. In Proceedings of the International Conference on Parallel Problem Solving from Nature, Coimbra, Portugal, 8-12 September 2018; pp. 424-435.

36. Tizhoosh, H.R. Opposition-based learning: A new scheme for machine intelligence. In Proceedings of the International Conference on Computational Intelligence for Modelling, Control and Automation and International Conference on Intelligent Agents, Web Technologies and Internet Commerce (CIMCA-IAWTIC'06), Vienna, Austria, 28-30 November 2005; Volume 1, pp. 695-701.

37. Shi, Y.; Eberhart, R. A modified particle swarm optimizer. In Proceedings of the 1998 IEEE International Conference on Evolutionary Computation Proceedings. IEEE World Congress on Computational Intelligence (Cat. No. 98TH8360), Anchorage, AK, USA, 4-9 May 1998; pp. 69-73. 
38. Liang, J.J.; Qin, A.K.; Suganthan, P.N.; Baskar, S. Comprehensive learning particle swarm optimizer for global optimization of multimodal functions. IEEE Trans. Evol. Comput. 2006, 10, 281-295. [CrossRef]

39. Chen, W.N.; Zhang, J.; Lin, Y.; Chen, N.; Zhan, Z.H.; Chung, H.S.H.; Li, Y.; Shi, Y.H. Particle swarm optimization with an aging leader and challengers. IEEE Trans. Evol. Comput. 2012, 17, 241-258. [CrossRef]

Publisher's Note: MDPI stays neutral with regard to jurisdictional claims in published maps and institutional affiliations.

(C) 2020 by the authors. Licensee MDPI, Basel, Switzerland. This article is an open access article distributed under the terms and conditions of the Creative Commons Attribution (CC BY) license (http:/ / creativecommons.org/licenses/by/4.0/). 